\title{
The prevalence of bipolar disorder in autoimmune disease: a systematic review and meta-analysis
}

\author{
Mengyi Chen, Qi Jiang, Lei Zhang \\ Department of Geriatrics, Shanghai Pudong New District Mental Health Center, Shanghai, China \\ Contributions: (I) Conception and design: M Chen; (II) Administrative support: M Chen; (III) Provision of study materials or patients: Q Jiang, L \\ Zhang; (IV) Collection and assembly of data: Q Jiang, L Zhang; (V) Data analysis and interpretation: M Chen; (VI) Manuscript writing: All authors; \\ (VII) Final approval of manuscript: All authors. \\ Correspondence to: Mengyi Chen. Shanghai Pudong New District Mental Health Center, Shanghai 200124, China. Email: 1141134986@qq.com.
}

Background At the end of the last century, genome-wide association studies revealed a significant
genetic association between bipolar disorder and autoimmune diseases. Subsequently, the theory of immune
pathogenesis of bipolar disorder gradually formed, and the research on autoimmune diseases and bipolar
comorbidities began to extend to other diseases, but their correlation is still controversial. To explore the
differences in the prevalence of bipolar disorder in patients with autoimmune disease and normal healthy
people through meta-analysis, and to examine the relationship between bipolar disorder and autoimmune
disease by reviewing the relevant literature. Methods: The Cochrane, PubMed, and Embase databases were searched by computer from the date of inception of the database to July 2020. The main topics of the search were based on common autoimmune diseases, including systemic lupus erythematosus, rheumatoid arthritis, psoriasis, multiple sclerosis, ulcerative colitis, Crohn's disease, ankylosing spondylitis, pemphigus, and Sjogren's syndrome. The databases were comprehensively searched for controlled studies regarding the prevalence of bipolar disorder in patients with autoimmune diseases. Review Manager 5.3 software was used for meta-analysis.

Results: In total, 10 cohort and case control studies were included. From these, 16 control groups were extracted based on nine autoimmune diseases. The meta-analysis demonstrated that the incidence of bipolar disorder was significantly increased in patients with autoimmune disease compared to patients without autoimmune disease, [mean difference $(M D)=1.54$, 95\% confidence interval $(\mathrm{CI}): 1.28-1.86, \mathrm{P}<0.00001$ ]. Also, in the meta-analysis based on five cross-sectional analyses (in which a total of five control groups were extracted based on five autoimmune diseases), the high comorbidity rate of autoimmune diseases and bipolar disorder was verified ( $\mathrm{MD}=2.23,95 \% \mathrm{CI}: 1.62-3.07, \mathrm{P}<0.00001)$.

Conclusions: The prevalence of bipolar disorder is markedly higher in patients with autoimmune disease. Yet, more basic research is needed to verify the special significance of immune mechanisms in bipolar disorder.

Keywords: Meta-analysis; autoimmune disease; bipolar disorder; comorbidity

Submitted Oct 20, 2020. Accepted for publication Dec 23, 2020.

doi: 10.21037/apm-20-2293

View this article at: http://dx.doi.org/10.21037/apm-20-2293

\section{Introduction}

Autoimmune disease is a general term for conditions that occur when the body attacks its own tissues due to an abnormally functioning immune system. The etiology and pathogenesis of autoimmune diseases are highly complex. It is generally believed that the immune tolerance of body tissues is destroyed or the immune balance function of the immune system is disturbed. Common autoimmune diseases often involve multiple systems and organs, and 
the prolonged course of illness and long treatment process often presents a considerable burden for patients. Bipolar disorder is a common psychiatric disorder, and although its treatment has become increasingly mature, its etiology remains unclear.

In recent years, the comorbidity between bipolar disorder and autoimmune disease has attracted the attention of researchers. A previous clinical questionnaire survey of patients with bipolar disorder found that the incidence of rheumatoid arthritis, thyroid disease, type 1 diabetes, and other immune-related diseases was significantly higher in this population (1). A larger controlled study involving structured interviews reported that patients with bipolar disorder had a significantly higher risk of multiple autoimmune diseases compared with patients in the control group, and that this risk was independent of the subtype of bipolar disorder (2). It has also been found that people with multiple autoimmune diseases are more likely to develop bipolar disorder than people with normal immune function. As early as 1987 , researchers found that the prevalence of bipolar disorder was significantly higher in patients with multiple sclerosis than in the general population $(\mathrm{P}<0.001)(3)$. Similar findings were also found in epidemiological surveys in different countries and regions $(4,5)$. At the end of the last century, genome-wide association studies revealed a significant genetic association between bipolar disorder and autoimmune diseases $(6,7)$. Subsequently, the theory of immune pathogenesis of bipolar disorder gradually emerged, and studies reporting on the comorbidity of autoimmune diseases and bipolar disorder began to extend to other diseases. In view of the high comorbidity rate between bipolar disorder and autoimmune diseases, as well as the high compatibility of the characteristics of both illnesses, some researchers have proposed that bipolar disorder is essentially an autoimmune disease. If this hypothesis is true, the treatment of bipolar disorder or autoimmune diseases should not be limited to one of their own, and the effects of the other must be taken into account in the treatment of one of them. In addition, the treatment methods and entry points for bipolar disorder will be updated. However, so far, we still can not come to a conclusion about the relationship between them.

The present study centered on several common autoimmune diseases with a: systemic lupus erythematosus, rheumatoid arthritis, psoriasis, multiple sclerosis, ulcerative colitis, Crohn's disease, ankylosing spondylitis, pemphigus, and Sjogren's syndrome. Ten cohort and case control studies from 2004 to the present (8-17) were summarized, 16 groups of comparative data were included, and a metaanalysis was performed. We present the following article in accordance with the PRISMA reporting checklist (available at http://dx.doi.org/10.21037/apm-20-2293).

\section{Methods}

\section{Literature search}

The Cochrane, PubMed, and Embase databases were comprehensively searched from the date of inception of the database to July 2020. A combination of the following keywords was used: ('autoimmune disease' OR 'systemic lupus erythematosus' OR 'rheumatoid arthritis' OR 'psoriasis' OR 'multiple sclerosis' OR 'ulcerative colitis' OR 'Crohn's disease 'OR' ankylosing spondylitis' OR 'pemphigus' OR' Sicca syndrome') AND ('manic depressive' OR 'bipolar disorder').

\section{Inclusion and exclusion criteria}

Studies were eligible for inclusion in this meta-analysis based on the following criteria: (I) published studies on the comorbidity of autoimmune disease and bipolar disorder; (II) cohort or case control study type; (III) complete data, where the original data could be extracted. Literature was excluded based on the following criteria: (I) reviews, case reports, and other non-academic literature; (II) incomplete data, or where original data could not be extracted; (III) studies lacking a normal control group, or the control group does not involve people without autoimmune disease. Data extraction and risk assessment of literature: the literature search, data extraction, and quality assessment were conducted independently by two doctors. In case of disagreement between the two doctors, a third doctor would re-evaluate the results. The entire screening and selection process are shown in Figure 1.

\section{Statistical analysis}

The International Classification of Diseases (ICD) and the Diagnostic and Statistic Manual of Mental Disorders (DSM) are the only two diagnostic criteria included, while other studies that do not describe the diagnostic criteria are not included in the study and analysis. In order to control confounders and reduce heterogeneity, information such as age, study type, diagnostic criteria, and race was extracted 


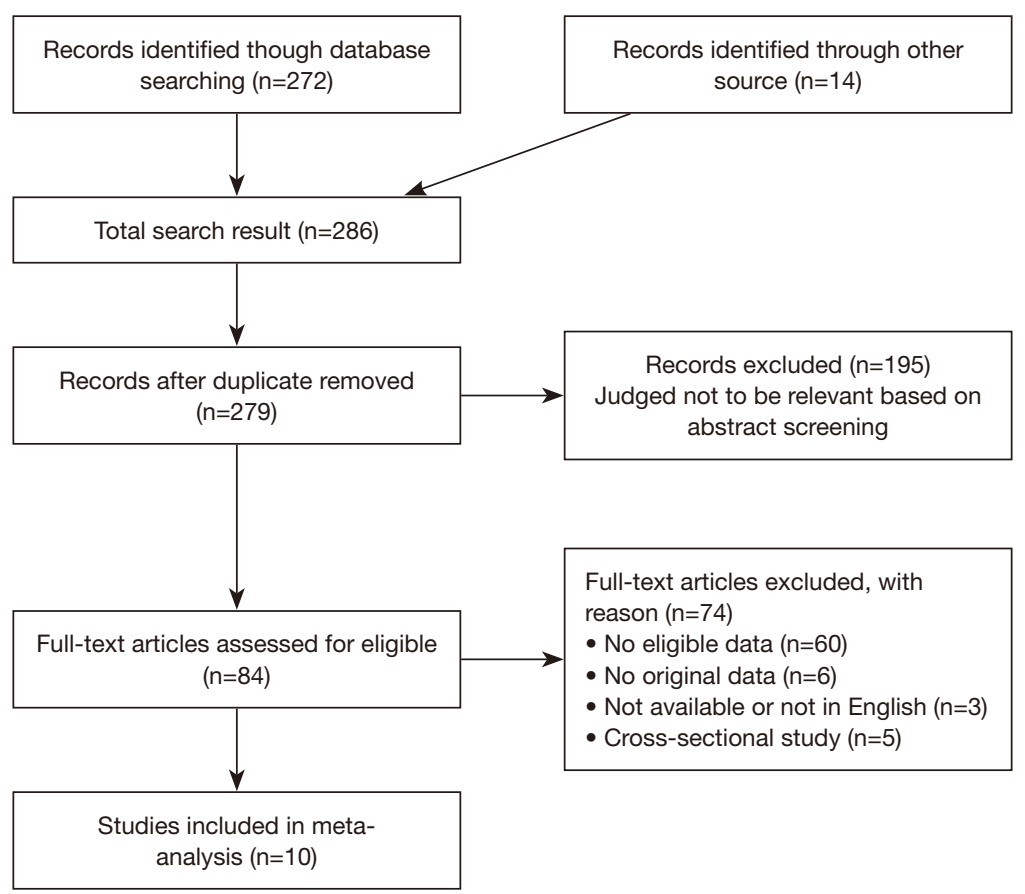

Figure 1 Study identification and literature review process.

from the included literature, and subgroup analyses were conducted based on the aforementioned factors (i.e., age, gender, study type, diagnostic criteria, and race). In addition, we also found five cross-sectional studies that reported on the comorbidity of bipolar disorder in patients with autoimmune diseases. Considering the limitations of a cross-sectional study design, we conducted a separate metaanalysis of these five studies.

Meta-analysis of data was performed using Review Manager 5.3 (https://community.cochrane.org/help/ tools-and-software/revman-5) Mean difference (MD) and $95 \%$ confidence interval (CI) were used for prevalence. Heterogeneity of the study was assessed by the heterotopic coefficient, $\mathrm{I}^{2}$. Heterogeneity between the included studies was determined by the $\mathrm{P}$ value and $\mathrm{I}^{2}$ test. $\mathrm{P}>0.1$ and $\mathrm{I}^{2}<50 \%$ indicated that there was no statistically significant heterogeneity among the studies, and in these cases, a fixed effects model was used for meta-analysis. However, if $\mathrm{P} \leq 0.1$ or $\mathrm{I}^{2} \geq 50 \%$, there was significant statistical heterogeneity among the studies, and a random effects model was used for meta-analysis. The source of heterogeneity and its influence on the stability of results were determined by sensitivity analysis. $\mathrm{P}<0.05$ was considered statistically significant. Funnel plots were used to assess publication bias.

\section{Results}

\section{Studies included in each meta-analysis}

Our literature search yielded 279 studies. A total of 195 studies were excluded after reading the titles and abstract, and 74 studies were excluded after reading the full text. Finally, 10 studies (8-17) were included in this meta-analysis. Among these, eight were cohort studies $(8,9,11-15,17)$ and two were case-control studies $(10,16)$. The data of the selected studies are shown in Table 1.

\section{Meta-analysis of the prevalence of bipolar disorder in patients with autoimmune disease}

Of the 10 included studies, one was related to Sicca syndrome $(8,9)$, one was related to systemic lupus erythematosus (9), one was related to inflammatory enteritis (Crohn's disease and ulcerative colitis) (9), four were related to rheumatoid arthritis (8-11), four were related to psoriasis (12-15), two were related to multiple sclerosis $(9,16)$, one was related to pemphigus (9), and one was related to ankylosing spondylitis (17). The sample sizes of these studies ranged from 1,005 to 933,880 . Our meta-analysis showed that the prevalence of bipolar disorder in patients 
Table 1 Summary of characteristics of studies in the current meta-analysis

\begin{tabular}{|c|c|c|c|c|c|c|c|c|}
\hline Study & Time & $\begin{array}{c}\text { Diagnostic } \\
\text { criteria }\end{array}$ & Diagnose & Research type & Mean age & $\begin{array}{c}\text { Gender } \\
\text { (female\%) }\end{array}$ & Country & Case/total \\
\hline \multirow[t]{2}{*}{ Hsieh } & 2019 & ICD-9 & SS & Cohort study & $53.2 \pm 13.6$ & 88.7 & China & $13 / 688$ \\
\hline & & & Control & & $53.2 \pm 13.6$ & 88.7 & & $55 / 3,440$ \\
\hline Wang & 2018 & ICD-9 & SS & Cohort study & NA & NA & China & $53 / 11,812$ \\
\hline \multirow[t]{2}{*}{ Wang } & 2018 & ICD-9 & SLE & Cohort study & NA & NA & China & $68 / 12,195$ \\
\hline & & & Control & & $46.4 \pm 19.5$ & 49.6 & & $697 / 261,992$ \\
\hline \multirow[t]{2}{*}{ Wang } & 2018 & ICD-9 & IE & Cohort study & NA & NA & China & $5 / 1,788$ \\
\hline & & & Control & & $46.4 \pm 19.5$ & 49.6 & & $697 / 261,992$ \\
\hline \multirow[t]{2}{*}{ Wang } & 2018 & ICD-9 & RA & Cohort study & NA & NA & China & $87 / 29,795$ \\
\hline & & & Control & & $46.4 \pm 19.5$ & 49.6 & & $697 / 261,992$ \\
\hline \multirow[t]{2}{*}{ Hsu } & 2014 & ICD-9 & $\mathrm{RA}$ & Cohort study & NA & NA & China & $32 / 2,570$ \\
\hline & & & Control & & NA & NA & & $15 / 2,570$ \\
\hline \multirow[t]{2}{*}{ Hsieh } & 2019 & ICD-9 & RA & Cohort study & $53.2 \pm 13.6$ & 88.7 & China & $18 / 1,302$ \\
\hline & & & Control & & $53.2 \pm 13.6$ & 88.7 & & $55 / 3,440$ \\
\hline Kimball & 2012 & ICD-9 & Ps & Cohort study & $11.4 \pm 4.1$ & 53.7 & U.S. & $26 / 7,404$ \\
\hline \multirow[t]{2}{*}{ Paller } & 2019 & ICD-9 & Ps & Cohort study & $12.9 \pm 3.2$ & 56.4 & China & $38 / 7,686$ \\
\hline & & & Control & & $12.9 \pm 3.2$ & 56.4 & & $80 / 30,744$ \\
\hline \multirow[t]{2}{*}{ Carta } & 2013 & DSM-IV & MS & Case control study & $38.9 \pm 10.0$ & 69.7 & Italy & $17 / 201$ \\
\hline & & & Control & & $38.8 \pm 10.1$ & 69.7 & & $2 / 804$ \\
\hline \multirow[t]{2}{*}{ Wang } & 2018 & ICD-9 & MS & Cohort study & NA & NA & China & $4 / 1,060$ \\
\hline & & & Control & & $46.4 \pm 19.5$ & 49.6 & & $697 / 261,992$ \\
\hline \multirow[t]{2}{*}{ Wang } & 2018 & ICD-9 & $\mathrm{Pe}$ & Cohort study & NA & NA & China & $0 / 945$ \\
\hline & & & Control & & $46.4 \pm 19.5$ & 49.6 & & $697 / 261,992$ \\
\hline \multirow[t]{2}{*}{ Shen } & 2016 & ICD-9 & AS & Cohort study & NA & 35.1 & China & $7 / 2,331$ \\
\hline & & & Control & & NA & 35.1 & & $14 / 9,324$ \\
\hline
\end{tabular}

BD, bipolar disorder; ICD, International Classification of Diseases; DSM, Diagnostic and Statistic Manual of Mental Disorders; SS, Sicca Syndrome; SLE, Systemic Lupus Erythematosus; IE, Inflammatory Enteritis (Ulcerative Colitis + Crohn Disease; RA, rheumatoid arthritis, Ps, psoriasis; MS, multiple sclerosis; Pe, pemphigus; AS, ankylosing spondylitis; NA, not available. 
with autoimmune disease was significantly higher compared to the control group ( $M D=1.54,95 \%$ CI: $1.28-1.86$, $\mathrm{P}<0.00001$ ) (Figure 2). The heterogeneity test indicated that there was heterogeneity in the included studies $\left(\mathrm{I}^{2}=72 \%\right)$, and a subgroup analysis was required to explore the source of this heterogeneity.

\section{Investigation of beterogeneity and publication bias}

The funnel plots of the 16 groups of data included in this meta-analysis are shown in Figure 3. The scatter points of the validity of each study are arranged in an inverted funnel shape symmetrically around the centerline, indicating that the publication bias in this meta-analysis is not significant.

\section{Subgroup meta-analyses}

\section{Subgroup meta-analysis of included studies based on} the ages of study participants

The included studies could be divided into two groups based on the ages of study participants, including a teenage group (8) (two groups of data) and an adult group $(12,15)$ (two groups of data). Other studies were not included in the subgroup analysis due to there being no restriction on the age of inclusion. The results showed that age was a source of heterogeneity. The prevalence of bipolar disorder was correlated with the ages of study participants and the prevalence of bipolar disorder in the teenage subgroup was higher than that in normal control group.

\section{Subgroup meta-analysis of the included studies based on the gender of study participants}

The included studies could be divided into two subgroups, male and female $(8,10,12-17)$. The results of the subgroup analysis showed that the gender was not the source of heterogeneity. However, the prevalence of bipolar disorder was related to the gender of participants, compared with the control group, the prevalence of bipolar disorder was higher in both male and female subgroups.

\section{Subgroup meta-analysis of included studies based on the study type}

The included studies could be divided into two groups based on the study type, including a cohort study group $(8,9,11-15,17)$ (14 groups of data) and a case control study group $(10,16)$ (two groups of data). The results of the subgroup meta-analysis demonstrated that study type was a source of heterogeneity. The cohort study type was correlated with the prevalence of bipolar disorder, and the prevalence of bipolar disorder in the cohort subgroup was higher than that in the control group.

\section{Subgroup meta-analysis of included studies based on the diagnostic criteria}

The included studies could be divided into two groups based on the diagnostic criteria, including an International Classification of Diseases (ICD) group $(8,9,11-15,17)$ (14 groups of data) and a DSM group (16) (one group of data). Other studies were not included in the subgroup analysis because the diagnostic criteria were not stated. The results demonstrated that diagnostic criteria were a source of heterogeneity. Both the ICD and DSM (as diagnostic criteria) were associated with the prevalence of bipolar disorder, and the prevalence of bipolar disorder in both subgroups was higher compared to the control group.

\section{Subgroup meta-analysis of included studies based on the race of study participants}

The included studies could be divided into three subgroups based on the races of study participants, including an Asian subgroup $(8-11,15,17)$ (12 groups of data), an American subgroup (12) (one group of data), and a European subgroup $(13,14,16)$ (three groups of data). The results of the subgroup analysis showed that participants' race is a source of heterogeneity. Asian and European participants were correlated with the prevalence of bipolar disorder, and the prevalence of bipolar disorder in these two subgroups was higher compared to the control group.

All subgroup analysis results are shown in Table 2.

\section{Meta-analysis of the cross-sectional studies}

In our literature search, we also discovered some crosssectional studies reporting on the comorbidity of bipolar disorder in patients with autoimmune disease, and so we carried out another meta-analysis based on these studies (18-22). Among the five included studies, one was related to systemic lupus erythematosus (18), one was related to inflammatory enteritis (Crohn's disease and ulcerative colitis) (19), two were related to multiple sclerosis $(20,21)$, and one was related to pemphigus (22). A total of 34,633 patients with autoimmune diseases and 238,200 controls were included. The results of the meta-analysis demonstrated that the prevalence of bipolar disorder in patients with autoimmune disease was considerably higher than compared to the control group ( $\mathrm{MD}=2.23,95 \% \mathrm{CI}$ : 


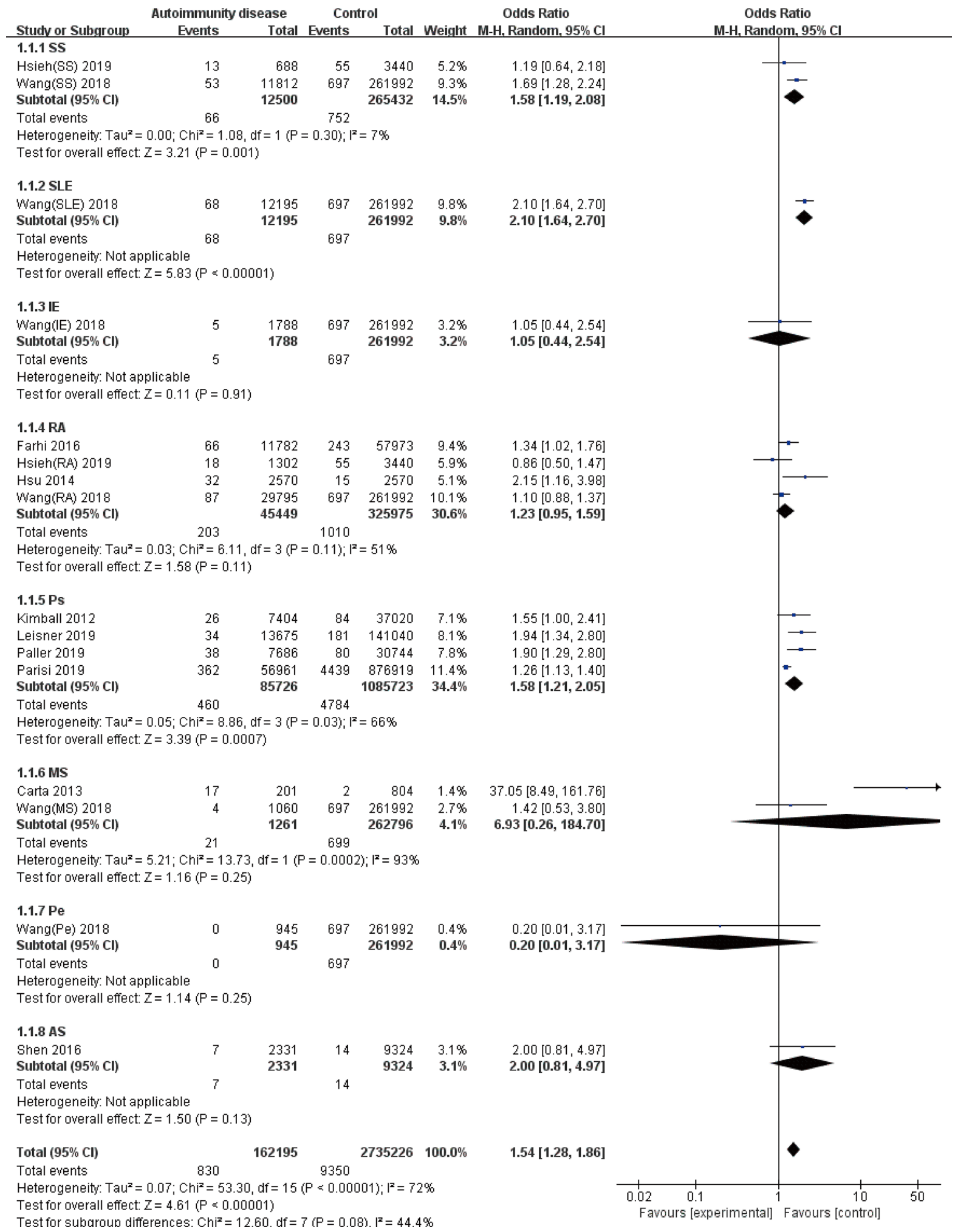

Figure 2 Meta-analysis of the prevalence of bipolar disorder in autoimmune disease patients. SS, Sicca Syndrome; SLE, Systemic Lupus Erythematosus; IE, Inflammatory Enteritis (Ulcerative Colitis + Crohn Disease); RA, rheumatoid arthritis, Ps, psoriasis; MS, multiple sclerosis; Pe, pemphigus; AS, ankylosing spondylitis. 


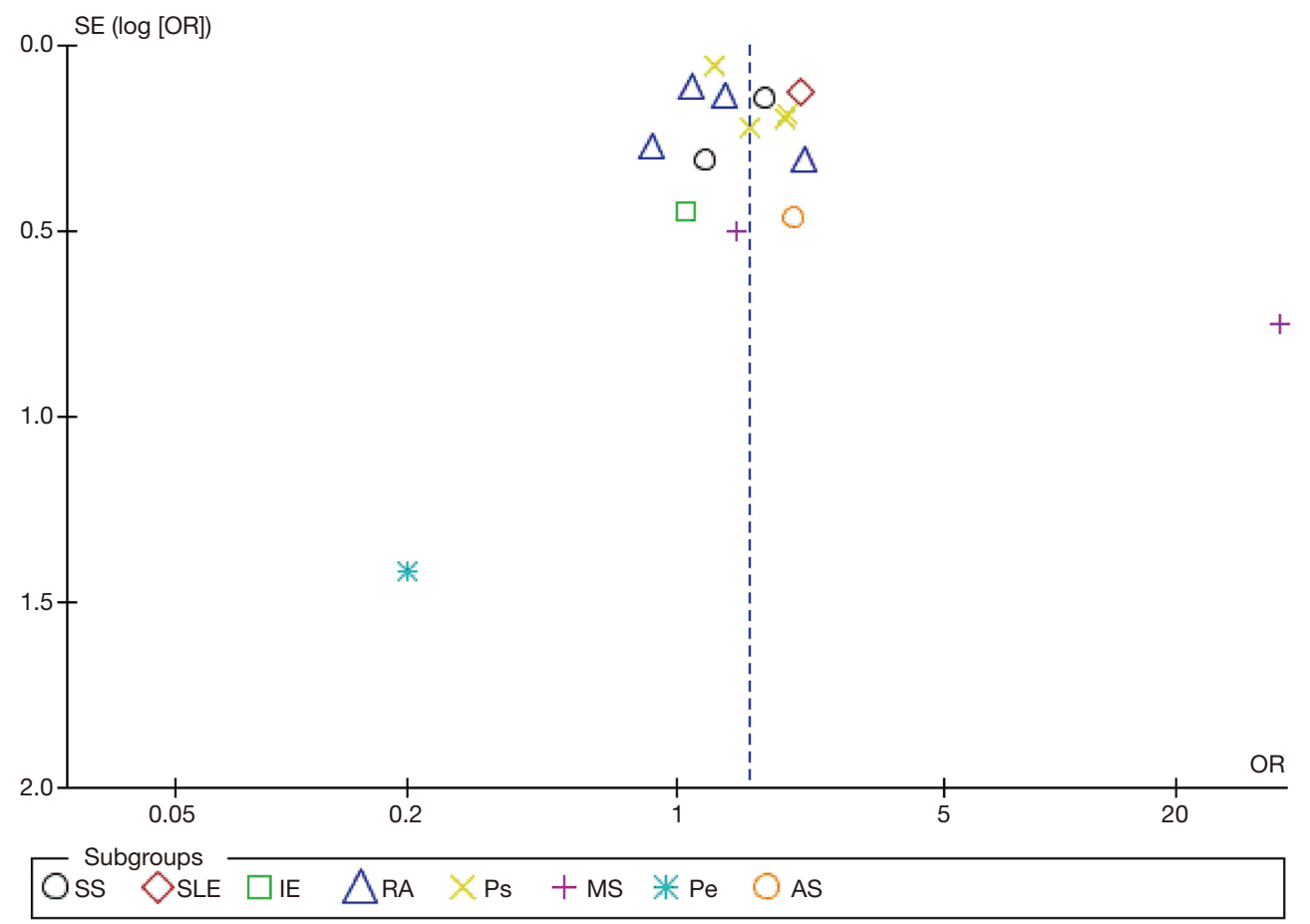

Figure 3 The funnel plot of this meta-analysis. SS, Sicca Syndrome; SLE, Systemic Lupus Erythematosus; IE, Inflammatory Enteritis (Ulcerative Colitis + Crohn Disease); RA, rheumatoid arthritis, Ps, psoriasis; MS, multiple sclerosis; Pe, pemphigus; AS, ankylosing spondylitis.

1.62-3.07, $\mathrm{P}<0.00001, \mathrm{I}^{2}=65 \%$ ) (Figure 4), which further validates the previous results.

\section{Discussion}

Bipolar disorder is a common, disabled, recurrent and severe mental health problem. It often occurs in late childhood or early adolescence. Symptoms include mania, hypomania, psychotic symptoms of depression in a relatively healthy period (23). The clinical course of bipolar disorder is changeable. Although bipolar disorder is defined by the presence of manic or manic symptoms, most patients spend most of their time in a state of depression, which is the main cause of disability (24). There are four subtypes of bipolar disorder: bipolar type I, bipolar type II, circulatory type and undefined type. Distinguish each subtype according to its characteristics, such as a description of the patient's current or recent attack. The rapid circulation feature is also applicable to bipolar type I or type II disorder. If the patient has at least 4 mood attacks in the first 12 months, the seizure is partially or completely relieved for at least 2 months or converted to a reverse episode (such as severe depressive episode to manic episode) (25). The human immune system is complex and dynamic, and our current understanding of it remains limited. Yet, existing studies have shown that bipolar disorder and the immune system exhibit a close and subtle link. Epidemiological investigation has shown that autoimmune thyroid disease is an independent risk factor for bipolar disorder, and this trait is more obvious in women (26). In our study, the prevalence of autoimmune diseases in patients with bipolar disorder was significantly higher than that in the control group, both male and female. These autoimmune diseases involve Sika syndrome, rheumatoid arthritis, psoriasis and ankylosing spondylitis. According to the U.S. Database of Child Hospitalizations, children hospitalized for autoimmune diseases have significantly higher rates of bipolar disorder comorbidity than those hospitalized for other reasons (27). Furthermore, in some exogenous allergy diseases, the high comorbidity rate of bipolar disorder cannot be ignored. Researchers followed tens of thousands of adolescents for 10 years and found that adolescents with exogenous allergy diseases (allergic rhinitis, allergic asthma, allergic dermatitis, and allergic conjunctivitis) were significantly more likely to 
Table 2 Subgroup analysis of incidence of bipolar disorder in patience with autoimmune disease

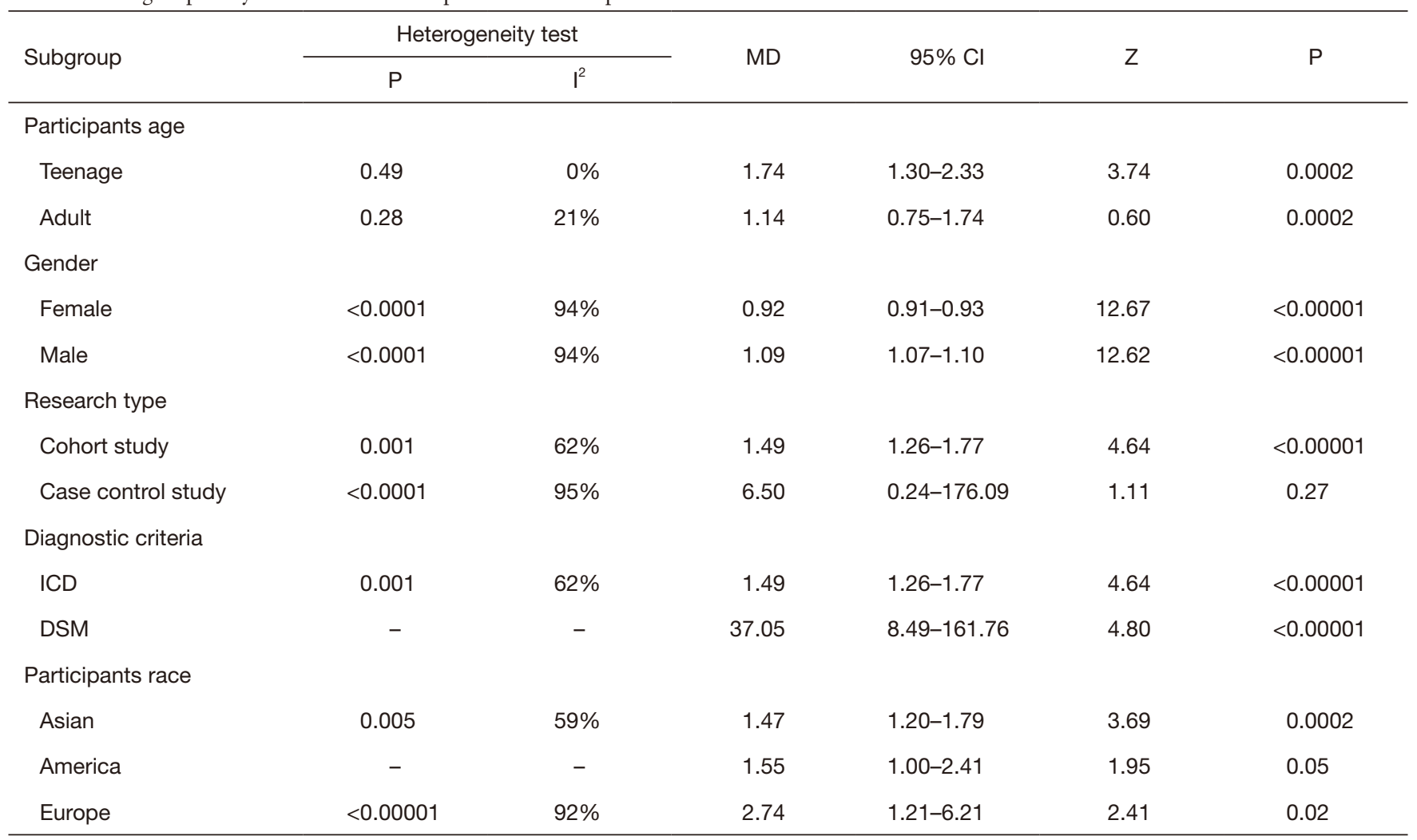

ICD, International Classification of Diseases; DSM, Diagnostic and Statistic Manual of Mental Disorders.

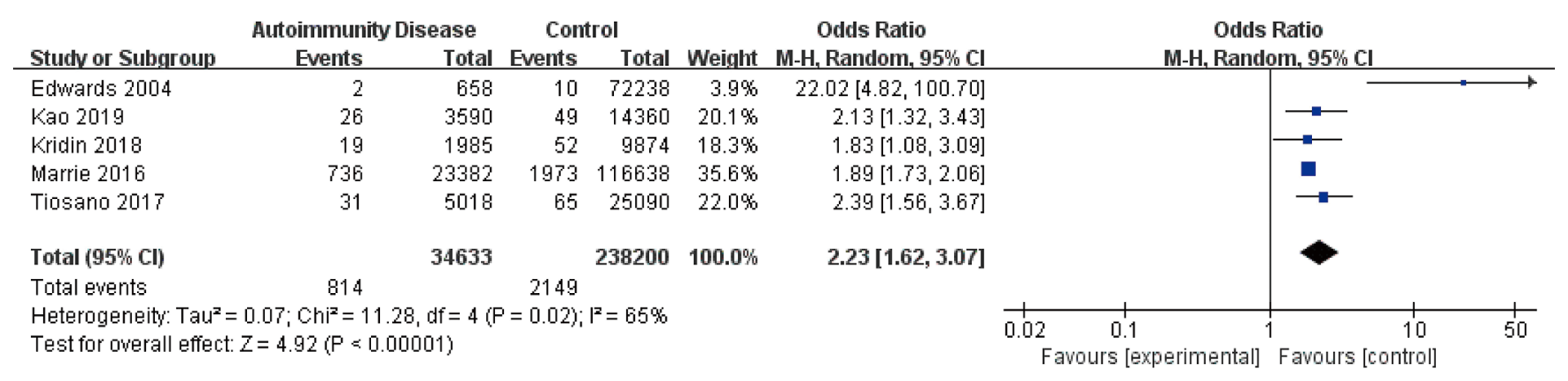

Figure 4 Meta-analysis of cross-sectional studies.

develop bipolar disorder in adulthood than those who had no history of exogenous allergy diseases. Also, the presence of multiple allergic diseases in adolescence leads to an even greater risk of developing bipolar disorder in adulthood (28). High comorbidities of bipolar disorder are also found in some of the rarer immune diseases. In a study of health data from the Danish population, people with a history of Guillain-Barre syndrome and autoimmune hepatitis were believed to have a notably increased risk of bipolar disorder (29). The impact of immune system dysfunction on the emotional system can even extend to the offspring of patients. Studies have shown that the children of pregnant women with abnormal thyroid function have a significantly increased risk of bipolar disorder (30).

The high prevalence of bipolar disorder in immune disease is not one-way, and previous studies have shown a marked increase in the prevalence of the immune disease in people with bipolar disorder. An epidemiological survey 
in the UK found that people with bipolar disorder had significantly increased rates of asthma, type 1 diabetes, and rheumatoid arthritis (31). A Swedish study of registered patients with schizophrenia and bipolar disorder reported considerable increases in the prevalence of rheumatoid arthritis, rheumatoid myalgia, and autoimmune thyroid disease in patients with bipolar disorder, while this feature not observed in the schizophrenia group (2). In a previous meta-analysis that simultaneously studied the prevalence of asthma in patients with bipolar disorder and the prevalence of bipolar disorder in asthma patients, the results showed that the prevalence of bipolar disorder in asthma patients was significantly higher compared to the normal control group, and the prevalence of asthma in patients with bipolar disorder was also higher compared to the normal control group (32). As the existing research believes that the human immune system is a whole, the presence of one immune disease will significantly increase the incidence of other immune diseases (33), which highlights the special significance of the immune system in bipolar disorder. Some researchers have proposed that bipolar disorder is essentially an autoimmune disease (34) and try to explain its pathogenesis by finding a specific antibody. In previous studies, researchers have found abnormal titers of various antibodies in patients with bipolar disorder (35), but some of these antibodies have corresponding relationships with certain known autoimmune diseases (36). Meanwhile, other antibodies are not very specific (37), and thus, they cannot establish a one-to-one relationship with bipolar disorder. Therefore, we cannot yet conclude that bipolar disorder is an autoimmune disease.

As a chronic disease with high rates of recurrence and disability, once diagnosed, bipolar disorder patients typically face long-term treatment and community health management. Bipolar disorder patients with an autoimmune disease comorbidity have a lower average life expectancy, a higher risk of self-injury, a higher readmission rate, more difficult treatment, and a higher risk of in-hospital death compared with bipolar disorder patients that do not have an autoimmune disease comorbidity (38-41). As a result, the treatment and management risk of such patients is more serious. The comorbidity of bipolar disorder and autoimmune disease can provide insights for future research and guide the treatment and management of the disease.

Firstly, in patients with bipolar disorder, there are a variety of abnormalities in the titers of non-specific antibodies, and most of these abnormalities occur before the initial onset of the disease (42). Therefore, immunological detection results have a role for the early warning of the onset of bipolar disorder in high-risk groups. Secondly, due to the variety of changes in the immunological index during the onset of bipolar disorder, such as the higher ratio of regulatory $T$ cells $(43,44)$, changes in the concentrations of various cytokines [interleukin (IL)-6, IL-8, IL-1 $\beta$, tumor necrosis factor (TNF)- $\alpha$, TNF- $\beta 1$, brain-derived neurotrophic factor (BDNF)] (45-47), as well as the degree of these changes, is associated with the severity of emotional disorders. Thus, these immunological indicators can also be used as a reference for the clinical treatment and outcomes of bipolar disorders. Thirdly, studies have shown that some non-specific immunological indicators (such as central granulocyte/lymphocyte ratio) can predict the suicide risk and cognitive level of patients with bipolar disorder $(48,49)$, which has practical significance for community health management and social function rehabilitation of patients. Finally, through investigation of the pathogenesis of autoimmune diseases, researchers have further speculated about the pathogenesis of bipolar disorder and proposed various mechanisms including cytokine-induced change of monoamine, increased oxidative stress, pathological excessive activation of microglia, excessive activation of the hypothalamus-pituitary-adrenal (HPA) axis, and immune disorder associated with sleep (45). At present, the inflammatory-emotional pathway has gradually become a new target for the treatment of bipolar disorder (50), and anti-inflammatory drugs and immunosuppressants for the treatment of bipolar disorder have gradually moved from concept to clinical trials. We look forward to further studies to evaluate the clinical effects of these therapies, so as to benefit the majority of patients as soon as possible.

\section{Conclusions}

The prevalence of bipolar disorder is significantly higher in patients with autoimmune disease. Yet, more basic research is needed to verify the special significance of immune mechanisms in bipolar disorder.

\section{Acknowledgments}

Funding: This study was funded by the Special Fund for Livelihood Research (Medical and Health Care) of the Science and Technology Development Fund of Pudong New District in 2020 (PKJ2020-Y33). 


\section{Footnote}

Reporting Checklist: The authors have completed the PRISMA reporting checklist. Available at http://dx.doi. org/10.21037/apm-20-2293

Conflicts of Interest: All authors have completed the ICMJE uniform disclosure form (available at http://dx.doi. org/10.21037/apm-20-2293). The authors have no conflicts of interest to declare.

Ethical Statement: The authors are accountable for all aspects of the work in ensuring that questions related to the accuracy or integrity of any part of the work are appropriately investigated and resolved.

Open Access Statement: This is an Open Access article distributed in accordance with the Creative Commons Attribution-NonCommercial-NoDerivs 4.0 International License (CC BY-NC-ND 4.0), which permits the noncommercial replication and distribution of the article with the strict proviso that no changes or edits are made and the original work is properly cited (including links to both the formal publication through the relevant DOI and the license). See: https://creativecommons.org/licenses/by-nc-nd/4.0/.

\section{References}

1. Forty L, Ulanova A, Jones L, et al. Comorbid medical illness in bipolar disorder. Br J Psychiatry 2014;205:465-72.

2. Cremaschi L, Kardell M, Johansson V, et al. Prevalences of autoimmune diseases in schizophrenia, bipolar I and II disorder, and controls. Psychiatry Res 2017;258:9-14.

3. Joffe RT, Lippert GP, Gray TA, et al. Mood Disorder and Multiple Sclerosis. Arch Neurol 1987;44:376-8.

4. Bozikas VP, Anagnostouli MC,Petrikis P, et al. Familial bipolar disorder and multiple sclerosis:a three-generation HLA family study. Prog Neuropsychopharmacol Biol Psychiatry 2003;27:835-9.

5. Marrie RA, Horwitz R, Cutter G, et al. The burden of mental comorbidity in multiple sclerosis: frequent, underdiagnosed and undertreated. Multiple Sclerosis 2009;15:385-92.

6. Kember RL, Hou L, Ji X, et al. Genetic pleiotropy between mood disorders, metabolic, and endocrine traits in a multigenerational pedigree. Transl Psychiatry 2018;8:218.

7. Tylee DS, Sun J, Hess JL, et al. Genetic correlations among psychiatric and immune-related phenotypes based on genome-wide association data. Am J Med Genet B Neuropsychiatr Genet 2018;177:641-57.

8. Hsieh MC, Hsu CW, Lu MC, et al. Increased risks of psychiatric disorders in patients with primary Sjögren's syndrome-a secondary cohort analysis of nationwide, population-based health claim data. Clin Rheumatol 2019;38:3195-203.

9. Wang LY, Chiang JH, Chen SF, et al. Systemic autoimmune diseases are associated with an increased risk of bipolar disorder: A nationwide population-based cohort study. J Affect Disord 2018;227:31-7.

10. Farhi A, Cohen AD, Shovman O, et al. Bipolar disorder associated with rheumatoid arthritis: A case-control study. J Affect Disord 2016;189:287-9.

11. Hsu CC, Chen SC, Liu CJ, et al. Rheumatoid arthritis and the risk of bipolar disorder: a nationwide population-based study. PLoS One 2014;9:e107512.

12. Kimball AB, Wu EQ, Guerin A, et al. Risks of developing psychiatric disorders in pediatric patients with psoriasis. Am Acad Dermatol 2012;67:651-7.e1-2.

13. Parisi R, Webb RT, Kleyn CE, et al. Psychiatric morbidity and suicidal behaviour in psoriasis: a primary care cohort study. Br J Dermatol 2019;180:108-15.

14. Leisner MZ, Riis JL, Schwartz S, et al. Psoriasis and Risk of Mental Disorders in Denmark. JAMA Dermatol 2019;155:745-7.

15. Paller AS, Schenfeld J, Accortt NA, et al. A retrospective cohort study to evaluate the development of comorbidities, including psychiatric comorbidities, among a pediatric psoriasis population. Pediatr Dermatol 2019;36:290-7.

16. Carta MG, Moro MF, Lorefice L, et al. The risk of Bipolar Disorders in Multiple Sclerosis. J Affect Disord 2014;155:255-60.

17. Shen CC, Hu LY, Yang AC, et al. Risk of Psychiatric Disorders following Ankylosing Spondylitis: A Nationwide Population-based Retrospective Cohort Study. J Rheumatol 2016;43:625-31.

18. Tiosano S, Nir Z, Gendelman O, et al. The association between systemic lupus erythematosus and bipolar disorder - a big data analysis. Eur Psychiatry 2017;43:116-9.

19. Kao LT, Lin HC, Lee HC. Inflammatory bowel disease and bipolar disorder: A population-based cross-sectional study. J Affect Disord 2019;247:120-4.

20. Edwards LJ, Constantinescu CS. A prospective study of conditions associated with multiple sclerosis in a cohort of 658 consecutive outpatients attending a multiple sclerosis clinic. Mult Scler 2004;10:575-81. 
21. Marrie RA, Patten SB, Tremlett H, et al. Sex differences in comorbidity at diagnosis of multiple sclerosis: A population-based study. Neurology 2016;86:1279-86.

22. Kridin K, Zelber-Sagi S, Comaneshter D, et al. Bipolar Disorder Associated with Another Autoimmune DiseasePemphigus: A Population-based Study. Can J Psychiatry 2018;63:474-80.

23. Grande I, Berk M, Birmaher B, et al. Bipolar disorder. Lancet 2016;387:1561-72.

24. Sole B, Jimenez E, Torrent C, et al. Cognitive Impairment in Bipolar Disorder: Treatment and Prevention Strategies. Int J Neuropsychopharmacol 2017;20:670-80.

25. Miller TH. Bipolar Disorder. Prim Care 2016;43:269-84.

26. Barbuti M, Carvalho AF, Kohler CA, et al. Thyroid autoimmunity in bipolar disorder: A systematic review. J Affect Disord 2017;221:97-106.

27. Sztein DM, Lane WG. Examination of the Comorbidity of Mental Illness and Somatic Conditions in Hospitalized Children in the United States Using the Kids' Inpatient Database. Hosp Pediatr 2016;6:126-34.

28. Wei HT, Lan WH, Hsu JW, et al. Risk of developing major depression and bipolar disorder among adolescents with atopic diseases: A nationwide longitudinal study in Taiwan. J Affect Disord 2016;203:221-6.

29. Eaton WW, Pedersen MG, Nielsen PR, et al. Autoimmune diseases, bipolar disorder, and non-affective psychosis. Bipolar Disord 2010;12:638-46.

30. Spann MN, Cheslack PK, Brown AS. The association of serologically documented maternal thyroid conditions during pregnancy with bipolar disorder in offspring. Bipolar Disord 2020;22:621-8.

31. Schoepf D, Heun R. Bipolar disorder and comorbidity: increased prevalence and increased relevance of comorbidity for hospital-based mortality during a 12.5year observation period in general hospital admissions. J Affect Disord 2014;169:170-8.

32. Wu MK, Wang HY, Chen YW, et al. Significantly Higher Prevalence Rate of Asthma and Bipolar Disorder CoMorbidity A Meta-Analysis and Review Under PRISMA Guidelines. Medicine (Baltimore) 2016;95:e3217.

33. Magyari M, Koch HN, Pfleger CC, et al. Gender and autoimmune comorbidity in multiple sclerosis. Mult Scler 2014;20:1244-51.

34. Pender MP. Hypothesis: bipolar disorder is an EpsteinBarr virus-driven chronic autoimmune disease implications for immunotherapy. Clin Transl Immunology 2020; 9:e1116.

35. Haggerty JJ, Evans DL, Golden RN, et al. The Presence of Antithyroid Antibodies in Patients with Affective and Nonaffective Psychiatric Disorders. Biol Psychiatry 1990;27:51-60.

36. Oomen HA, Schipperijn AJ, Drexhage HA. The prevalence of affective disorder and in particular of a rapid cycling of bipolar disorder in patients with abnormal thyroid function tests. Clin Endocrinol (Oxf) 1996;45:215-23.

37. Leon CJ, Pacchiarotti I, Murru A, et al. Bipolar disorder and antibodies against the $\mathrm{N}$-methyl-d-aspartate receptor: A gate to the involvement of autoimmunity in the pathophysiology of bipolar illness. Neurosci Biobehav Rev 2015;5 5:403-12.

38. Dickerson F, Origoni A, Schroeder J, et al. Mortality in schizophrenia and bipolar disorder: Clinical and serological predictors. Schizophr Res 2016;170:177-83.

39. Singhal A, Ross J, Seminog O, et al. Risk of self-harm and suicide in people with specific psychiatric and physical disorders: comparisons between disorders using English national record linkage. J R Soc Med 2014;107:194-204.

40. Thomsen AF, Kvist TK, Andersen PK, et al. Increased risk of affective disorder following hospitalisation with hyperthyroidism - a register-based study. Eur J Endocrinol 2005;152:535-43.

41. Chebli S, Zgueb Y, Ouali U, et al. Bipolar Disorder as Comorbidity with Sjogren's Syndrome: What Can We Do? Case Rep Psychiatry 2020; 2020:8899615.

42. Benedetti F, Aggio V, Pratesi ML, et al. Neuroinflammation in Bipolar Depression. Front Psychiatry 2020;11:71.

43. Drexhage RC, Hoogenboezem TH, Versnel MA, et al. The activation of monocyte and $\mathrm{T}$ cell networks in patients with bipolar disorder. Brain Behav Immun 2011;25:1206-13.

44. Kuwabara T, Matsui Y, Ishikawa F, et al. Regulation of T-Cell Signaling by Post-Translational Modifications in Autoimmune Disease. Int J Mol Sci 2018;19:819.

45. Rosenblat JD, McIntyre RS. Bipolar Disorder and Immune Dysfunction: Epidemiological Findings, Proposed Pathophysiology and Clinical Implications. Brain Sci 2017;7:144.

46. Chen M, Zhang L, Jiang Q. Peripheral IGF-1 in bipolar disorder and major depressive disorder: a systematic review and meta-analysis. Ann Palliat Med 2020;9:4044-53.

47. Luo $\mathrm{Y}, \mathrm{He} H$, Zhang $M$ et al. Altered serum levels of TNF- $\alpha$, IL- 6 and IL-18 in manic, depressive, mixed state of bipolar disorder patients. Psychiatry Res 2016;244:19-23.

48. Ivkovic M, Pantovic SM, Dunjic KB, et al. Neutrophilto-lymphocyte ratio predicting suicide risk in euthymic patients with bipolar disorder: Moderatory effect of family history. Compr Psychiatry 2016;66:87-95. 
49. Sağlam Aykut D, Arslan FC, Karagüze EO, et al. The relationship between neutrophil-lymphocyte, plateletlymphocyte ratio and cognitive functions in bipolar disorder. Nord J Psychiatry 2018;72:119-23.

50. SayuriYamagata A, Brietzke E, Rosenblat JD, et al. Medical

Cite this article as: Chen M, Jiang Q, Zhang L. The prevalence of bipolar disorder in autoimmune disease: a systematic review and meta-analysis. Ann Palliat Med 2021;10(1):350-361. doi: 10.21037/apm-20-2293 comorbidity in bipolar disorder: The link with metabolicinflammatory systems. J Affect Disord 2017;211:99-106.

(English Language Editor: A. Kassem) 